\title{
Sélection et stabilité du rendement chez le blé tendre d'hiver
}

\author{
M Brancourt-Hulmel ${ }^{1 *}, \mathrm{C}^{\text {Lecomte }}{ }^{2}$ \\ avec la participation technique de $M$ Leleu $^{1}, P$ Bérard ${ }^{3}, N$ Galic ${ }^{4}, B$ Trouvé 5 , C Sausseau 6 \\ 1 INRA, domaine de Brunehaut, F80200 Estrées-Mons; \\ 2 INRA, domaine d'Époisses, Bretenières, F21110 Genlis \\ 3 INRA, domaine de Crouelle, F63036 Clermont-Ferrand cedex ; \\ ${ }^{4}$ Station de génétique végétale, ferme du Moulon, F91120 Gif-sur-Yvette ; \\ 5 INRA, domaine de la Minière, F78280 Guyancourt; \\ ${ }_{6}$ INRA, station d'amélioration des plantes, centre de recherche de Rennes, BP 29, F35650 Le Rheu, France
}

(Reçu le 11 juillet 1994; accepté le 3 janvier 1995)

\begin{abstract}
Résumé - Douze lignées de blé tendre en cours de sélection à l'INRA et 3 témoins ont été étudiés pour la stabilité du rendement dans le réseau multilocal INRA. Ce matériel a été évalué dans des dispositifs à 2 blocs randomisés, les parcelles élémentaires totalisant au moins $5,9 \mathrm{~m}^{2}$. Au total, 24 milieux diversifiés ont été étudiés durant les campagnes 1990-1991, 1991-1992 et 1992-1993. Le facteur milieu a été défini par la combinaison des niveaux des facteurs lieu, mode cultural et année. Dans chaque milieu, une description biologique a été réalisée pendant les phases de mise en place du nombre de grains par $\mathrm{m}^{2}$ et de remplissage du grain. L'analyse de la stabilité a pris en compte sous forme de covariables les caractéristiques du milieu définies au cours de la description précédente et a permis ainsi de constater que le comportement des génotypes se différencie uniquement pendant le remplissage. Par ailleurs, les 2 modèles d'analyse de l'interaction, régression conjointe et régression factorielle, ont permis de préciser que le comportement des lignées INRA est bien différent de celui des témoins. En effet, les génotypes sélectionnés à Rennes sont, à l'inverse des témoins, les plus résistants aux conditions défavorables de milieu. Deux autres lignées sélectionnées à Clermont-Ferrand et à Estrées-Mons sont à la fois productives et stables, présentant une bonne adaptabilité générale et un assez bon niveau de résistance aux conditions défavorables de milieu, ce qui laisse présager qu'il est possible d'associer rusticité et productivité en sélection.
\end{abstract}

\section{blé tendre d'hiver / stabilité / interaction / rendement / sélection}

Summary - Breeding and yield stability of winter wheat. The yield stability of 12 lines of winter wheat bred by INRA (Institut National de Recherche Agronomique) and 3 controls has been studied in the INRA network. The material was evaluated in randomized 2-block designs, elementary plots measuring at least $5.9 \mathrm{~m}^{2}$. Twenty-four various environments have been studied over 3 seasons: 1990-1991, 1991-1992 and 1992-1993. They are combinations of levels of 3 factors: location, condition and year. Each environment has been biologically described during the period of the establishment of the number of grains per square meter and the grain-filling period. The analysis of stability included environmental characteristics as covariates. These were defined in a previous description and showed that the geno-

\footnotetext{
* Correspondance et tirés à part
} 
types only behaved in a different way during the grain-filling period. In addition, the 2 models used for the analysis of interaction, joint regression and factorial regression showed that the behaviour of the INRA lines was quite different from that of controls. In contrast to controls, the genotypes selected in Rennes are the most resistant to unfavourable conditions of the environment. Two other entries, selected in Clermont-Ferrand and Estrées-Mons, are simultaneously productive, stable, with good general adaptability and a rather good resistance to unfavourable conditions of environment, which indicates that it is possible to associate hardiness and yield potential in a breeding programme.

winter wheat / stability / interaction / yield / breeding

\section{INTRODUCTION}

La stabilité du rendement est un critère important pour le développement d'une variété. Parmi la multitude de termes employés, les termes de stabilité phénotypique, de stabilité du rendement et d'adaptation ou d'adaptabilité sont le plus souvent utilisés avec des sens parfois différents. On peut avancer d'ores et déjà qu'il n'y a pas de définition unique de la stabilité. La stabilité phénotypique caractérise l'importance des fluctuations du rendement observées pour un même génotype cultivé dans différents milieux. Ces fluctuations dépendent des effets des milieux et des interactions génotype*milieu. L'absence de définition unique est due au fait que lorsqu'on analyse l'interaction génotype ${ }^{*}$ milieu, seulement une partie de la variation des interactions peut être expliquée par les méthodes décomposant l'interaction. Toutes les définitions qui vont suivre recouvrent ce sens de stabilité phénotypique. Becker et Leon (1988) opposent le concept statique de la stabilité au concept dynamique.

Derrière le concept statique se cachent plusieurs synonymes : stabilité de type I (Lin et al, 1986), homéostasie et concept biologique (Becker, 1981). Un génotype stable présente une faible variance due aux milieux.

Le concept dynamique repose sur toutes les procédures qui permettent de quantifier l'interaction génotype ${ }^{\star}$ milieu et, quelle que soit la procédure, le matériel utilisé influence les résultats (Becker et Leon, 1988). II recouvre donc le concept agronomique de stabilité (Becker, 1981), l'adaptation ou l'adaptabilité et les différents types de stabilité (II, III et IV) définis par Lin et al (1986, 1988).

Le concept agronomique fait intervenir des paramètres de stabilité de type II. Un génotype stable réagit parallèlement à la réponse moyenne des génotypes avec lesquels il est testé. Cette stabilité est définie selon la qualité d'ajustement des données au modèle additif. Parmi les paramètres statistiques rencontrés pour décrire cette stabilité, on trouve l'écovalence variétale (Wricke,
1962), paramètre que Becker (1981) a pris en exemple pour définir le concept agronomique. II s'agit de la contribution du génotype à la somme totale des carrés des écarts de l'interaction. De la même façon, on peut définir une écovalence du milieu (Parisot-Baril, 1992).

La stabilité de type III est reliée à l'adaptation ou l'adaptabilité : un génotype stable présente un faible écart par rapport à la régression sur l'index du milieu (moyenne de toutes les lignées du milieu). Cette définition est donc reliée à la qualité d'ajustement selon le modèle de régression conjointe. Plusieurs auteurs ont décrit et élaboré cette méthode de régression : Yates et Cochran (1938), Finlay et Wilkinson (1963), Eberhart et Russell (1966) et Perkins et Jinks (1968). Selon Bilbro et Ray (1976), la stabilité est mesurée par le coefficient de détermination, c'est-à-dire le rapport entre la variation expliquée par le modèle et la variation totale. Le coefficient de régression est une mesure de l'adaptation ou de l'adaptabilité. On oppose l'adaptation spécifique à une région, à certains types de milieux à l'adaptation générale qui désigne un génotype adapté à une large gamme de milieux. On parle aussi de génotypes spécialistes et de génotypes généralistes. Selon Becker (1981), cette méthode de régression peut être assimilée à une combinaison des concepts biologique et agronomique de stabilité dans la mesure où les coefficients de régression sont très bien corrélés aux variances du milieu (concept biologique) et que les déviations à la régression le sont très bien aux écovalences (concept agronomique). Par ailleurs, Denis $(1980,1988)$ a généralisé cette méthode à travers l'utilisation de covariables associées aux génotypes et aux milieux : la régression factorielle.

Un dernier type de stabilité, le type IV défini par Lin et al en 1988, fait intervenir le facteur temps. La variation du milieu est séparée en une composante prévisible due à l'interaction génotype*lieu et une composante imprévisible due à l'interaction génotype*année. Cette dernière permet une mesure de la stabilité ou de la capacité 
d'un cultivar à résister à des variations imprévisibles qui sont en effet causées par les effets années. Pour ces 2 derniers types (III et IV), la stabilité ou plus exactement l'instabilité est donc mesurée par les irrégularités imprévisibles du génotype dans la réponse aux environnements.

À ces méthodes paramétriques, s'ajoutent des méthodes non paramétriques de mesure de la stabilité (Hühn, 1990a, b ; Hühn et al, 1993) dont certaines sont très corrélées aux précédentes (Piepho et al, 1992). Toutefois, toutes ces approches donnent seulement des caractéristiques individuelles de la stabilité mais ne fournissent pas une image globale de la réponse. En outre, un génotype stable selon un des critères ne l'est pas forcément selon les autres. Ceci provient du fait que les réponses des génotypes aux conditions d'environnement sont multivariables alors que les analyses précédentes sont univariables. L'analyse de régression factorielle précédemment décrite répond à ce dilemme ainsi que de nombreuses autres méthodes (Freeman, 1973 ; Lin et al, 1986 ; Westcott, 1986 ; Becker et Leon, 1988 ; Gauch, 1988, 1990). L'analyse en composantes principales (PCA), le modèle AMMI (Additive main effects and multiplicative interaction) qui associe à la fois des composantes additives et multiplicatives et les méthodes de classification sont les plus utilisés.

À l'INRA (Institut national de la recherche agronomique), la stabilité et l'adaptation des lignées de blé tendre d'hiver (Triticum aestivum L) sélectionnées par différents laboratoires de l'institut sont évaluées lors de l'expérimentation multilocale. Nous présenterons les résultats (stabilité de types II et III) de 12 lignées et 3 témoins étudiés dans 24 milieux très diversifiés qui ont tous fait l'objet d'un diagnostic agronomique.

\section{MATÉRIEL ET MÉTHODES}

\section{Description du réseau}

Le matériel de base de cette étude est constitué par des lignées de blé tendre expérimentées au sein du réseau multilocal INRA. Ce réseau, créé à partir des années 1970, permet d'expérimenter chaque année les lignées sélectionnées par l'INRA. À l'issue de cette expérimentation multilocale et pluriannuelle, les meilleures lignées sont déposées pour l'inscription au catalogue officiel des variétés. Ce réseau est subdivisé en 2 grandes zones climatiques: la zone Nord et la zone Sud. II existe également 2 séries : la série B correspond aux lignées entrant dans le réseau pour la première année et la série A comprend les lignées qui commencent leur deuxième, voire troisième, année d'expérimentation et qui ont montré de bons résultats de rendement et de stabilité à la récolte précédente.

Les essais de série $B$ sont cultivés selon un seul mode traité aux fongicides. Représentatif des pratiques culturales de la région d'expérimentation, ce mode permet de mesurer la productivité. Les essais de série $A$ sont conduits selon au moins 2 modes culturaux par site pour révéler des facteurs limitants du milieu. Le système fonctionne donc par éliminations successives et chaque année environ $70 \%$ des lignées sont éliminées. Le nombre important de lignées expérimentées en première année oblige à les répartir dans plusieurs essais et ainsi il existe en général 2 à 3 essais de série $B$ pour un seul essai de série $A$ par année.

\section{Données expérimentales}

L'étude a concerné les 3 dernières campagnes du réseau Nord : 1990-1991, 1991-1992, 1992-1993. Quinze lignées, toutes communes aux 3 années, ont été retenues pour l'étude. Elles constituent un sousensemble des lignées qui ont été expérimentées durant les 3 années puisque environ $70 \%$ du matériel est éliminé à l'issue de chaque année d'expérimentation (voir paragraphe précédent). Par ailleurs, elles pouvaient se trouver dans des essais différents alors que les témoins étaient présents dans tous les essais. Outre les 3 témoins, Apollo, Soissons et Thésée, on trouve donc:

- 2 lignées de I'INRA de Clermont-Ferrand : CF9104 et CF9107 ;

- 2 lignées de l'INRA de Dijon : DI9003 et DI9105;

- 5 lignées de l'INRA de Rennes : RE9006, RE9009, RE9102, RE9103 et RE8914;

- 3 lignées de l'INRA d'Estrées-Mons (le travail de sélection avait débuté en collaboration avec l'INRA de Versailles) : VM9014, VM9017 et VM9112.

Les témoins sont des variétés figurant au catalogue officiel français: Thésée a été inscrite en 1983, Apollo en 1986 et Soissons en 1987.

Les lignées INRA sont en cours de sélection. En 1991 , lors de leur première année de mise en expérimentation multilocale, les lignées de Dijon et d'Estrées-Mons étaient à la génération F6-F7 et celles de Clermont et Rennes à la génération F7-F8. En 1994, certaines d'entre elles sont présentées à l'inscription au catalogue français des variétés ou susceptibles de l'être.

Les lieux étudiés sont ceux pour lesquels une description biologique du milieu a été réalisée : les 4 stations INRA de Clermont-Ferrand, Dijon, Estrées-Mons et Rennes, la station de génétique végétale du Moulon et l'ancien domaine INRA de La Minière. Le descriptif de ces différents lieux, sols et modes culturaux est indiqué dans le tableau $\mathrm{I}$.

Le facteur milieu a été défini par la combinaison des niveaux des facteurs lieu, mode et année afin d'utiliser 
Tableau I. Descriptif des sites expérimentaux entrant dans l'étude.

Lieu

Sols

1
Modes culturaux

\section{2}

3

Clermont (63) Limons volcaniques Non traité aux fongicides Traité aux fongicides

Dijon (21)

Sols argilo-limoneux sur marnes et graviers

Semis tardif

Semis précoce

Estrées-Mons (80)

Limons profonds

Non traité aux fongicides

Traité aux fongicides

Idem mode 2 et battants

sur autre précédent

La Minière (78) Limons profonds

Non traité aux fongicides Traité aux fongicides

Le Moulon (91)

Limons profonds argileux

Non traité aux fongicides

Traité aux fongicides

Rennes (35)

Limons légers

Non traité aux fongicides

Traité aux fongicides

les modèles statistiques modélisant l'interaction entre 2 facteurs (Denis, 1992).

Au total, chaque lignée a été testée dans 24 conditions de milieu. Le nombre de modes culturaux a été variable entre les années et entre les lieux (tableau II) : les lignées entrant en première année (en 1991) dans les essais de série $B$ du réseau multilocal ont été expérimentées selon un seul mode traité aux fongicides (mode 2 dans le tableau I) et à partir de leur deuxième année d'expérimentation dans les essais de série $A, 2$ à 3 modes culturaux ont été pris en compte. Le mode 1 vise à faire apparaître des contraintes du milieu : attaques parasitaires pour les conditions sans fongicide, froid hivernal sur les plantules pour le semis tardif à Dijon. À Estrées-Mons, les modes 2 et 3 ont été implantés sur des précédents très répandus en Picardie, respectivement les précédents de pois de conserve (ou haricot selon les années) et de betteraves sucrières.

Le dispositif commun à tous les milieux comprenait 2 blocs randomisés. Tous les essais ont été semés à une densité de 300 grains par $\mathrm{m}^{2}$. La surface de la parcelle élémentaire était variable d'un lieu à un autre et comprise entre 6 et $7,5 \mathrm{~m}^{2}$ (tableau III).

Une description biologique de chaque milieu a été réalisée à l'aide de 4 variétés de référence, Arminda, Camp-Rémy, Soissons et Talent. Elle est basée sur l'analyse de l'élaboration du rendement de ces 4 témoins pendant la phase végétative et le remplissage (Lecomte et al, 1993). Chacune de ces phases est caractérisée par une variable «bilan»: le nombre de grains par $\mathrm{m} 2$ pour la première et le poids de mille grains pour la deuxième. La comparaison à une valeur de référence permet de révéler la présence d'un facteur limitant au cours de la phase, s'il existe un déficit par rapport à la référence. Celle-ci est définie, pour le poids de mille grains, par la meilleure valeur enregistrée lors d'expérimentations antérieures et, pour le nombre de grains, par la valeur qui a permis d'atteindre le rendement le plus élevé lors de ces mêmes expérimentations. Le tableau IV présente les valeurs de référence pour le rendement, le poids de mille grains et le nombre de grains par $\mathrm{m}^{2}$ des 4 variétés témoins utilisées dans l'étude. Deux covariables

Tableau II. Milieux intervenant dans l'étude de l'interaction.

Lieu

1991

Mode 2

Clermont (63)

Dijon (21)

Estrées-Mons (80)

La Minière (78)

Le Moulon (91)

Rennes (35)

$\begin{array}{ll} & \\ x & x \\ x & x \\ x & x\end{array}$

1993

992

Mode 1 Mode 2

Mode 3

$\begin{array}{cccc} & & & \\ \mathrm{x} & \mathrm{x} & \mathrm{x} & \\ \mathrm{x} & \mathrm{x} & \mathrm{x} & \mathrm{x} \\ \mathrm{x} & \mathrm{x} & \mathrm{x} & \\ \mathrm{x} & \mathrm{x} & \mathrm{x} & \end{array}$


Tableau III. Descriptif des parcelles pour les différents lieux.

\begin{tabular}{|c|c|c|c|c|c|}
\hline Lieu & $\begin{array}{l}\text { Longueur } \\
\qquad(m)\end{array}$ & $\begin{array}{l}\text { Largeur } \\
\qquad(m)\end{array}$ & Nombre de rangs & $\begin{array}{l}\text { Écartement entre rangs } \\
\qquad(m)\end{array}$ & $\begin{array}{c}\text { Surface } \\
\left(m^{2}\right)\end{array}$ \\
\hline & $\ldots$ & $\ldots$ & $--\quad \square$ & -- & \\
\hline Clermont (63) & 5 & 1,30 & 6 & 0,20 & 6,5 \\
\hline Dijon (21) & 6 & 1,25 & 6 & 0,18 & 7,5 \\
\hline Estrées-Mons (80) & 5 & 1,30 & 6 & 0,20 & 6,5 \\
\hline La Minière (78) & 6 & 1,30 & 6 & 0,18 & 7,8 \\
\hline Le Moulon (91) & 4 & 1,50 & 6 & 0,23 & 6,0 \\
\hline Rennes (35) & 5 & 1,30 & 6 & 0,20 & 5,9 \\
\hline
\end{tabular}

Tableau IV. Références des témoins pour le rendement et ses composantes.

\begin{tabular}{|c|c|c|c|}
\hline Variété & $\begin{array}{c}\text { Rendement maximum } \\
\left(q / \text { ha à } 15 \%{ }^{*}\right)\end{array}$ & PMG potentiel ( $g$ à $15 \%{ }^{*}$ ) & Nombre de grains par $m^{2}$ \\
\hline & -- & $-\quad-\quad-$ & $-\cdots \cdots$ \\
\hline Arminda & 123,2 & 46,0 & 28000 \\
\hline Camp Rémy & 120,0 & 46,2 & 26500 \\
\hline Soissons & 136,3 & 47,0 & 29000 \\
\hline Talent & 111,5 & 45,0 & 27500 \\
\hline
\end{tabular}

PMG : poids de 1000 grains ; * s'applique à la teneur en eau.

sont ensuite créées : la variable réduction ou déficit du nombre de grains et la variable réduction du poids de mille grains. Elles ont été utilisées comme covariables du milieu dans l'étude ultérieure de l'interaction génotype*milieu.

Pour identifier la nature et l'intensité du facteur limitant intervenu dans un essai, des indicateurs du milieu (indicateurs climatiques ou des notations de verse et de maladies) ont été associés à ces variables. À partir de ces indicateurs, de nouvelles covariables ont été

Tableau V. Liste des covariables du milieu.

\section{Période levée-floraison}

Déficit du nombre de grains par $\mathrm{m}^{2}$

Froid hivernal

Gel d'épi

Faible luminosité à la méiose

Nombre de jours secs

Déficit hydrique

Intensité de verse *

Pression d'oïdium *

Pression des autres maladies du feuillage *

Pression des maladies du pied * définies selon la méthode décrite par Lecomte et al (1993) et utilisées pour l'analyse de l'interaction génotype* milieu (tableau V).

\section{Analyses statistiques}

Du fait de la répartition des lignées dans plusieurs essais en 1991, les données de rendement en grain
Période de remplissage

Déficit du poids de mille grains

Déficit hydrique

Températures supérieures à $25^{\circ} \mathrm{C}$

Intensité de verse *

Pression d'oïdium *

Pression de septoriose *

Pression des autres maladies du feuillage *

Pression des maladies de l'épi *

Pression des maladies du pied *

* Notée sur les témoins du diagnostic agronomique. 
ont été corrigées par l'effet essai à partir des 3 témoins communs aux différents essais. Par ailleurs, elles ont aussi été corrigées par un effet bloc estimé à partir de toutes les lignées communes aux 2 blocs d'un milieu. En outre, seul un des 4 essais a été utilisé en 1991 pour les témoins afin de ne pas créer de déséquilibre par rapport aux autres lignées : en effet les lignées ont été observées sur 2 répétitions dans chaque milieu et les témoins sur $4^{\star} 2$ répétitions. Nous avons pu montrer que les résultats obtenus dans la partie Résultats et discussion étaient identiques quel que soit l'essai retenu pour les témoins.

Des écovalences variétales (Wricke, 1962) ont été calculées (paramètres de stabilité de type II). En ce qui concerne les modèles, outre les modèles additif et interactif complet, l'analyse de l'interaction génotype ${ }^{*}$ milieu pour la variable rendement en grain a été également réalisée à l'aide d'un modèle de régression conjointe (Finlay et Wilkinson, 1963) et des modèles de régression factorielle, développés par Denis $(1980,1988)$ et appliqués pour l'analyse du blé tendre d'hiver par Baril (1992) et Parisot-Baril (1992). Dans ces derniers modèles, les covariables du milieu sont issues de la description biologique. Ces modèles de régression ont permis l'estimation des paramètres de stabilité de type III.

Les traitements statistiques ont été réalisés à l'aide des logiciels INTERA version 3.3 (Decoux et Denis, 1991) et SAS version 6.07 (SAS Institute, 1991) et les différents modèles statistiques étaient de la forme :

$$
\begin{gathered}
E\left[X_{i j}\right]=\mu+\alpha_{i}+\beta_{j} \text { pour le modèle additif } \\
E\left[X_{i j}\right]=\mu+\alpha_{i}+\beta_{j}+\theta_{i j} \text { pour le modèle interactif complet } \\
E\left[X_{i j}\right]=\mu+\alpha_{i}+\beta_{j}+\lambda_{j} \hat{\beta}_{j} \text { pour la régression conjointe } \\
E\left[X_{i j}\right]=\mu+\alpha_{i}+\beta_{j}+\rho, c_{j} \text { pour la régression factorielle }
\end{gathered}
$$

où $\mu=$ moyenne générale, $\alpha_{i}=$ effet principal génotype, $\beta_{j}=$ effet principal milieu, $\theta_{i j}=$ interaction génotype ${ }^{\star}$ milieu, $\lambda_{i}=$ terme de régression génotypique sur la covariable $\hat{\boldsymbol{\beta}}_{j}, \mathrm{c}_{j}=$ covariable du milieu, $\rho_{i}=$ terme de régression génotypique sur la covariable $c_{j}$.

Enfin nous avons procédé à l'analyse de 4 regroupements différents :

- regroupement 1 : tous les milieux (24 au total) ;

- regroupement $2: 13$ milieux traités aux fongicides représentatifs des conduites culturales régionales;

- regroupement 3 : les 13 milieux précédents plus 4 milieux traités aux fongicides présentant une variante sur la date de semis ou le précédent cultural ;

- regroupement $4: 7$ milieux non traités aux fongicides.

\section{RÉSULTATS ET DISCUSSION}

\section{Importance de l'interaction}

Les rendements moyens pour les regroupements 1 à 4 sont respectivement de $84,2 \mathrm{q} / \mathrm{ha}, 89,4$ $\mathrm{q} / \mathrm{ha}, 88,3 \mathrm{q} / \mathrm{ha}$ et $74,2 \mathrm{q} / \mathrm{ha}$. L'analyse du modèle interactif complet effectuée pour la variable rendement en grain met en évidence une interaction génotype*milieu significative dans tous les regroupements (tableau VI). Quel que soit le regroupement, le coefficient de variation est identique et atteint la valeur de $4,8 \%$, ce qui indique une bonne qualité des regroupements et des essais.

Par comparaison des coefficients de détermination associés au modèle additif et interactif complet, on peut observer que l'interaction représente environ $20 \%$ de la variation totale de la somme des carrés d'écarts pour les 3 premiers regroupements (tableau $\mathrm{VII}$ ). On constate que le regroupement des milieux non traités aux fongicides présente une interaction de $28 \%$ qui est légèrement supérieure à celle des regroupements précédents.

Ces niveaux d'interaction sont assez élevés par comparaison à ceux obtenus par ParisotBaril (1992) dans une étude réalisée sur le blé tendre d'hiver. Avec des lignées expérimentées sur le même réseau que le nôtre, elle a trouvé des valeurs inférieures ou égales à $20 \% 6$ années sur 8 .

\section{Analyse des écovalences}

L'observation des écovalences génotypiques montre que les génotypes ne présentent pas les mêmes niveaux de stabilité (fig 1). Cinq génotypes dont l'écovalence est inférieure à $5 \%$ pour la plupart des regroupements apparaissent plus stables que les autres : il s'agit de CF9107, RE9006, RE8914, VM9017 et VM9112. Bien que le regroupement 4 des milieux, n'ayant pas reçu de fongicides, ait tendance à exacerber les différences, les résultats sont comparables d'un regroupement à un autre. Apollo et Thésée, 2 des 3 témoins, apparaissent comme les plus interactifs. Globalement, on constate donc que les lignées en cours de sélection semblent plus stables que les témoins et elles le sont d'autant plus que les milieux sont plus diversifiés (regroupements 1 et 4 ).

\section{Ajustement selon le modèle de régression conjointe}

La décomposition de l'interaction par un modèle de régression conjointe, utilisant l'effet principal milieu comme covariable, explique environ $25 \%$ 
Tableau VI. Tableaux d'analyse de variance pour les 4 regroupements : modèle interactif complet.

Sources de variation

Regroupement 1

Milieu

Génotype

Milieu * génotype

Erreur
$S C E$

$\begin{array}{rr}80383,1 & 23 \\ 7814,3 & 14 \\ 25891,3 & 322\end{array}$

5825,1

$d d l$

$\begin{array}{rr}23 & 3494,9 \\ 14 & 558,2 \\ 322 & 80,4 \\ 360 & 16,2\end{array}$

$C M$
$F$

$\operatorname{Pr}>F$

$R^{2}=0,95 ; \mathrm{CV}=0,048 ; \sigma_{\mathrm{e}}=4,0 \mathrm{q} / \mathrm{ha} ;$ moyenne $: 84,2 \mathrm{q} / \mathrm{ha}$

\section{Regroupement 2}

Milieu

Génotype

Milieu * génotype

Erreur

$\begin{array}{rr}32521,9 & 12 \\ 6250,5 & 14 \\ 9685,4 & 168 \\ 3588,0 & 195\end{array}$

2710,2
446,5
57,7
18,4

216,0

0,0001

0,0001

0,0001

\section{Regroupement 3 \\ Milieu \\ Génotype \\ Milieu * génotype \\ Erreur}

\begin{tabular}{rrrr}
34940,1 & 16 & 2183,8 & 123,4 \\
8111,7 & 14 & 579,4 & 32,7 \\
11109,3 & 224 & 49,6 & 2,8 \\
4513,4 & 255 & 17,7 & \\
\multicolumn{4}{r}{$R^{2}=0,92 ; C V=0,048 ; \sigma_{e}=4,2 q /$ ha $;$ moyenne $: 88,3 q /$ ha }
\end{tabular}

Regroupement 4
Milieu
Génotype
Milieu * génotype
Erreur

$\begin{array}{rr}16075,2 & \\ 5681,2 & 14 \\ 8803,4 & 84 \\ 1311,6 & 105\end{array}$

$R^{2}=0,96 ; \mathrm{CV}=0,048 ; \sigma_{\mathrm{e}}=3,5 \mathrm{q} / \mathrm{ha} ;$ moyenne $: 74,2 \mathrm{q} / \mathrm{ha}$

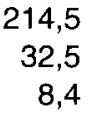

0,0001

0,0001

0,0001

Tableau VII. Comparaison des $R^{2}$ (en \%) pour les modèles additifs et interactifs complets.

\section{Regroupement 1}

73,5

95,1

1

\section{Regroupement 2}

74,5

93,1
Regroupement 3

0,0001

0,0001

0,0001
Modèle additif

Modèle interactif de la somme des carrés d'écart de l'interaction pour les regroupements 1 et 4 (tableau VIII), c'est-à-dire ceux faisant intervenir des milieux diversifiés. Bien que cette décomposition soit significative pour les 2 autres regroupements, seulement $10 \%$ de l'interaction sont expliqués : dans des gammes de milieux comparables et ayant reçu des modes culturaux équivalents, les lignées sélectionnées à l'INRA se comportent de la même façon alors qu'elles se différencient plus les unes des autres dans des gammes de milieux diversifiés.

Les résultats relatifs à cette méthode varient donc selon les milieux. Ils varient aussi selon les espèces : sur soja, Gauch (1988) a trouvé 9\% tandis que Finlay et Wilkinson (1963) ont obtenu $79 \%$ sur orge. Bien que cette méthode ne soit pas toujours efficace pour expliquer l'interaction, 


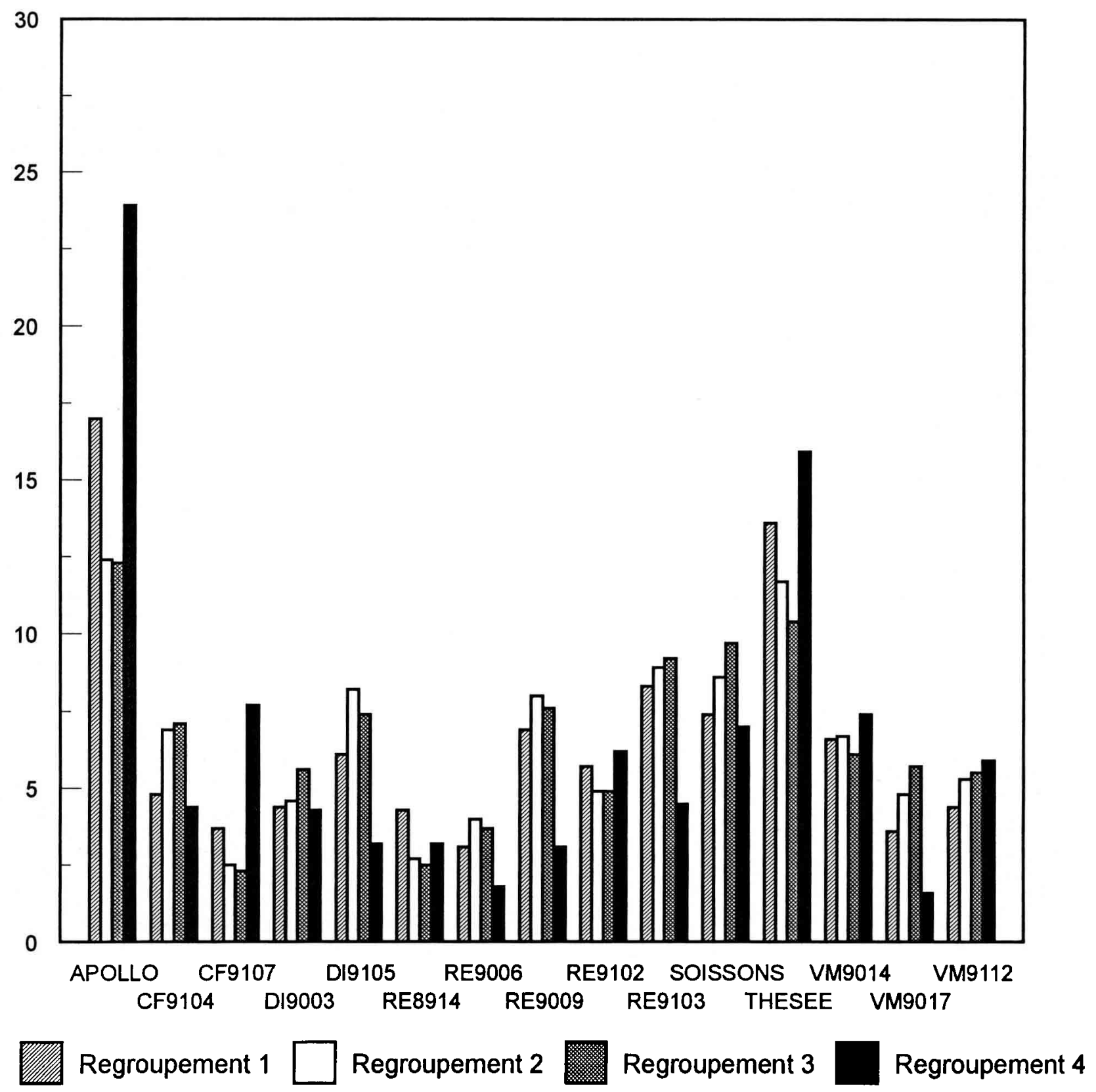

Fig 1. Écovalences génotypiques en \% pour les 4 regroupements.

Becker et Leon (1988) pensent qu'elle est intéressante pour étudier les génotypes aux comportements extrêmes. Ce sera l'objet des paragraphes suivants.

L'estimation des coefficients de régression variétaux associés à ce modèle permet de constater qu'ils sont cohérents avec les valeurs d'écovalence : en effet, à l'exception de RE8914, les génotypes les plus stables, dont l'écovalence est inférieure à $5 \%$, sont aussi ceux pour lesquels le coefficient de régression est proche de 1 tandis que les fortes valeurs d'écovalence sont associées à des coefficients très différents de 1 (fig 2). Suggérée par un coefficient de corrélation linéaire de 0,55 (significatif au seuil $\alpha=$ 0,05 ), cette assez bonne concordance des 2 paramètres ne se retrouve pas forcément chez d'autres espèces. Becker en 1981 (cité par Becker et Leon, 1988) constate une corrélation significative $(0,36)$ entre l'écovalence et le coefficient de régression chez l'avoine alors qu'il n'en trouve pas pour le maïs ou pour l'orge. 
Tableau VIII. Tableaux d'analyse de variance pour les 4 regroupements : régression conjointe.

Sources de variation

Regroupement 1

Milieu * génotype

Régression conjointe

Reste interaction

Erreur
Regroupement 2
Milieu * génotype
Régression conjointe
Reste interaction
Erreur

\section{Regroupement 3 \\ Milieu * génotype \\ Régression conjointe \\ Reste interaction \\ Erreur}

\section{Regroupement 4 \\ Milieu * génotype \\ Régression conjointe \\ Reste interaction \\ Erreur}

SCE

$d d l$

25891,3
5796,4
20094,8
5825,1

322

14

308

360

9685,4

1157,7

8527,8

3588,0

168

14

154

195

11109,3

1070,8

10038,4

4513,4

224

14

210

255

8803,4

2279,5

6523,8

1311,6
$C M$

$F$

$\operatorname{Pr}>F$

SCE/SCE interaction

$\begin{array}{rl}5,0 & 0,0001 \\ 25,6 & 0,0001 \\ 4,0 & 0,0001\end{array}$

3,1

4,5

3,0

55,4

18,4

0,0001

0,0001

$\begin{array}{ll}2,8 & 0,0001 \\ 4,3 & 0,0001 \\ 2,7 & 0,0001\end{array}$

8,4

13,0

7,5
0,0001

0,0001

0,0001
$22,4 \%$

$12,0 \%$

$9,6 \%$

$25,9 \%$
Ces coefficients traduisent aussi l'adaptabilité génotypique définie par Bilbro et Ray (1976). II est à noter que seuls les 5 génotypes de Rennes, dont RE8914, montrent une valeur inférieure à 1 et comprise entre 0,57 et 0,86 (tableau $(X)$ : ce sont des génotypes qui ont bien valorisé les milieux défavorables, notamment les milieux n'ayant pas reçu de traitements fongicides : ils ont présenté une adaptation spécifique à ces milieux tandis que leur rendement a été moyen dans des milieux où les pressions de maladies ont été beaucoup plus faibles. À l'inverse, Apollo et Thésée ont des coefficients très supérieurs à 1 et leur moyenne peu élevée indique que ces génotypes se sont mal comportés en milieu défavorable et n'ont exprimé un bon rendement que lorsque les milieux sont devenus favorables. Ils ont donc présenté une adaptabilité spécifique aux milieux favorables. La figure 3 , où seuls RE8914 et Apollo sont portés en exemple, illustre ces différences de comportement entre les génotypes de Rennes et ces 2 témoins. Sur la figure 4 , on constate que CF9107 et VM9112, et VM9017 dans une moindre mesure, associent productivité et adaptabilité générale. Par ailleurs, ils possèdent une bonne stabilité (fig 2). Sur cette même figure, on constate qu'il n'y a pas de relation linéaire entre la productivité et les coeffi-
93,2

12,5 cients de régression : le coefficient de corrélation atteint seulement 0,16 .

II apparaît que les milieux n'ayant pas reçu de traitement fongicide discriminent bien les génotypes résistants aux maladies de ceux qui ne le sont pas, car les génotypes les mieux adaptés à ces derniers ont en effet présenté les meilleures notes de résistance aux différentes maladies. $\mathrm{Si}$ ces milieux sont efficaces pour améliorer l'adaptation spécifique des génotypes, il est toutefois nécessaire d'y associer des milieux productifs. Ceci rejoint la proposition de Simmonds (1991) selon laquelle la sélection doit s'établir dans des environnements contrastés bien que ce genre de sélection appliqué à l'orge soit controversé par Sage et al (1984).

Mesurer la stabilité d'un génotype est utile en sélection. Pour améliorer l'adaptation générale du blé tendre de printemps, Braun et al (1992) ont démontré que les environnements les plus discriminants étaient les milieux n'ayant pas eu de stress abiotique ou biotique à l'exception des rouilles brune et jaune. Chez différentes céréales à paille, la sélection directe dans des milieux défavorables est la plus efficace pour améliorer la productivité dans de tels environnements (Ceccarelli, 1987 ; Atlin et al, 1989 ; Ceccarelli, 1989). Ceci est basé sur le fait que la sélection 
coefficients de régression

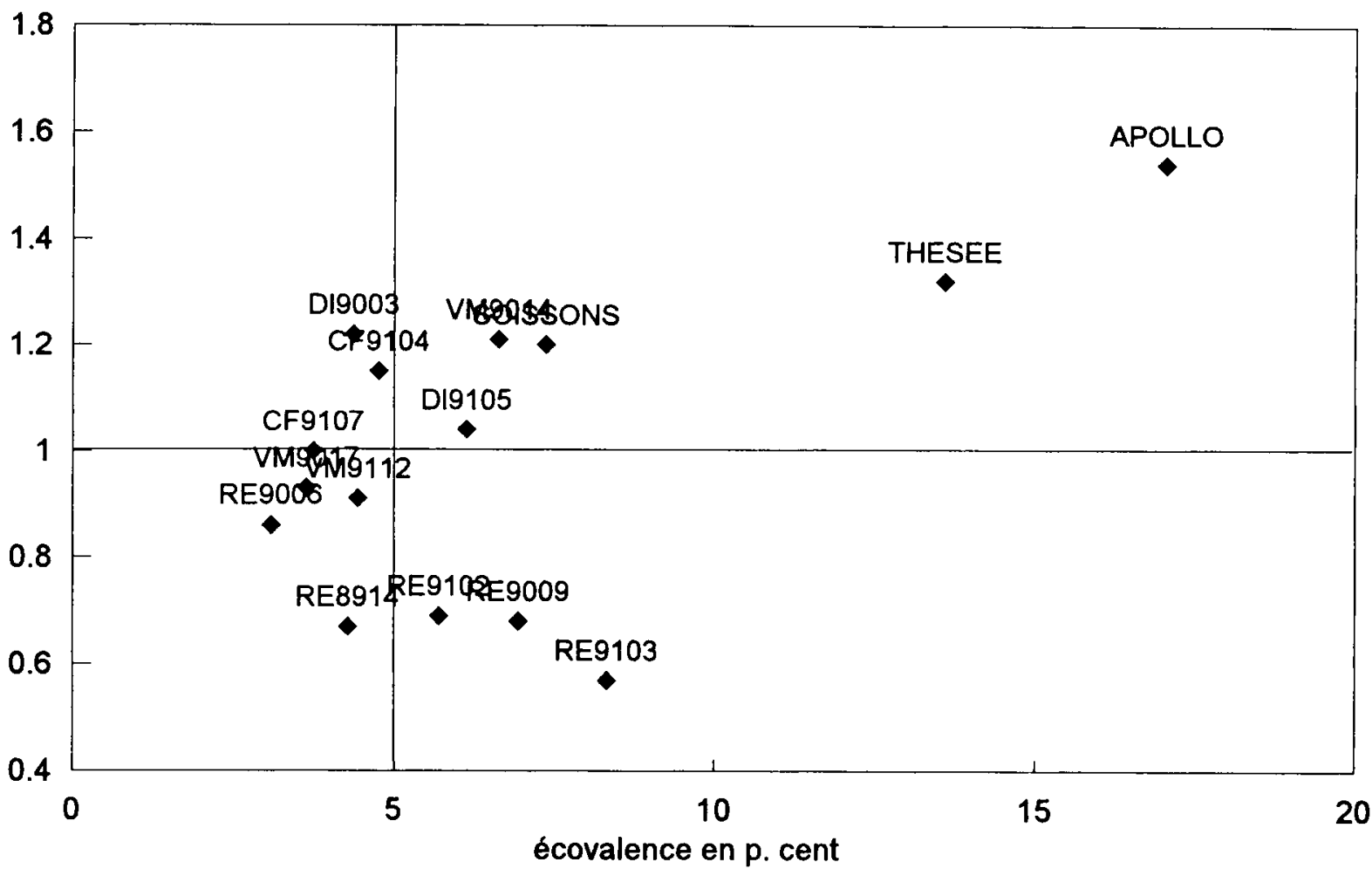

Fig 2. Modèle de régression conjointe : coefficients génotypiques de régression en fonction des écovalences pour le regroupement 1.

Tableau IX. Coefficients génotypiques de régression et $R^{2}$ (en \%) pour le regroupement 1 : modèle de régression conjointe.

\begin{tabular}{|c|c|c|}
\hline Variété & Coefficient & $\mathrm{R}^{2}$ \\
\hline Apollo & 1,54 & 81,9 \\
\hline Soissons & 1,20 & 82,0 \\
\hline Thésée & 1,32 & 75,9 \\
\hline CF9104 & 1,15 & 86,4 \\
\hline CF9107 & 1,00 & 84,8 \\
\hline DI9003 & 1,22 & 90,2 \\
\hline DI9105 & 1,04 & 78,4 \\
\hline RE8914 & 0,67 & 82,1 \\
\hline RE9006 & 0,86 & 85,1 \\
\hline RE9009 & 0,68 & 66,5 \\
\hline RE9102 & 0,69 & 72,6 \\
\hline RE9103 & 0,57 & 59,9 \\
\hline VM9014 & 1,21 & 84,3 \\
\hline VM9017 & 0,93 & 83,6 \\
\hline VM9112 & 0,91 & 80,2 \\
\hline
\end{tabular}

dans des environnements productifs ne produit pas de réponse ou une réponse négative dans des environnements peu productifs (Ceccarelli et al, 1992).

\section{Ajustement selon le modèle de régression factorielle}

À l'aide d'un modèle de régression factorielle, I'utilisation de covariables du milieu décrivant la période levée-floraison et le remplissage permet d'analyser plus en détail ces différences de comportement.

En ce qui concerne la période levée-floraison, la covariable du milieu associée explique assez peu l'interaction (environ $8 \%$ quel que soit le regroupement) : les 15 génotypes ont tendance à se comporter de la même façon durant cette période (tableau $X$ ).

C'est pendant le remplissage et pour les regroupements faisant intervenir des milieux diversifiés qu'apparaissent les différences (tableau X) : la covariable décrivant le remplissage représente $26 \%$ de l'interaction pour le premier regroupement et $37 \%$ pour le quatrième. Les autres milieux différencient moins les géno- 
rendement moyen du génotype en $q /$ ha

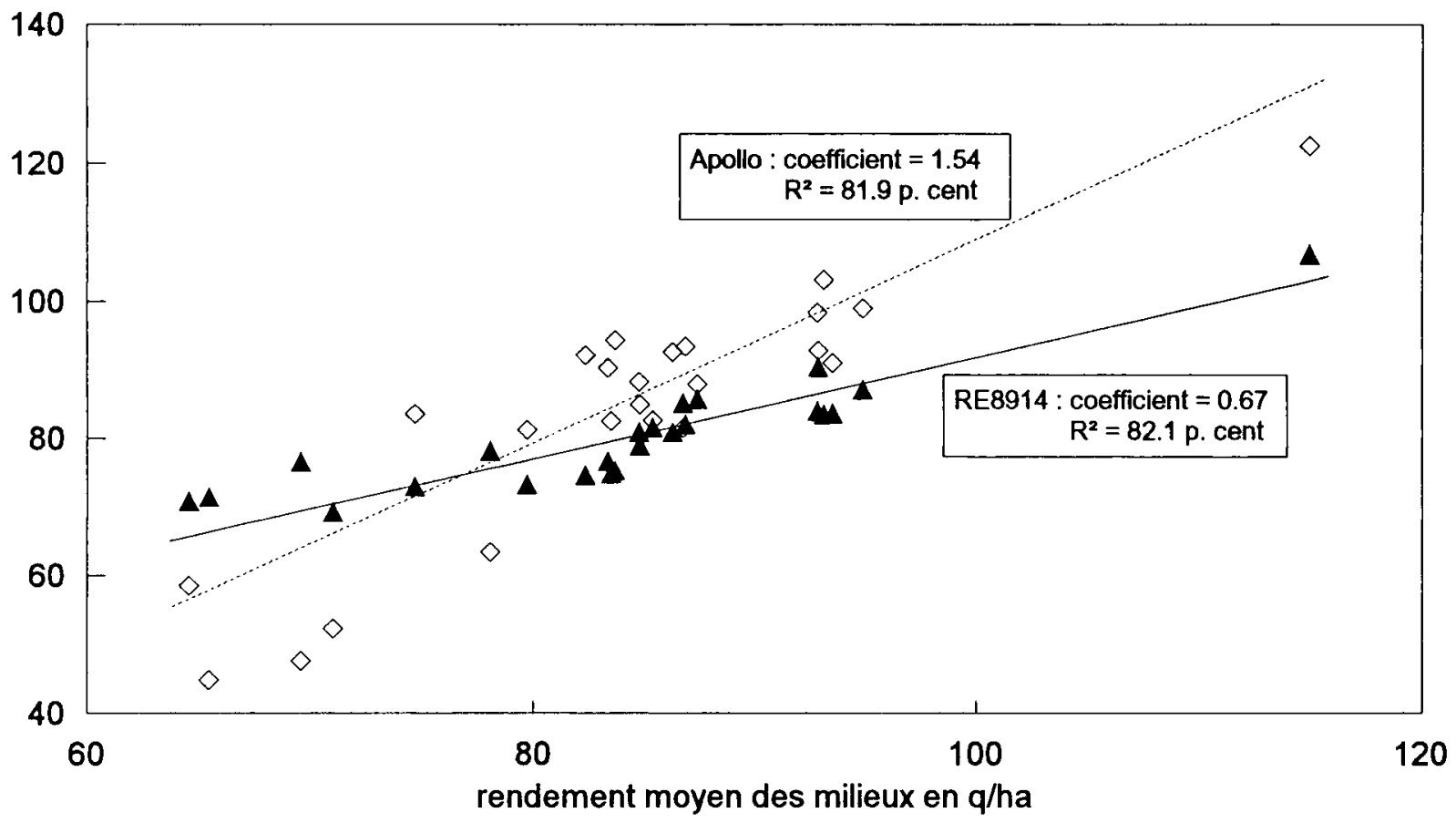

$\diamond$ Apollo (observé) \ RE8914 (observé)

Fig 3. Modèle de régression conjointe : courbe de réponse de 2 génotypes de blé tendre aux rendements moyens des milieux.

Tableau X. Pourcentage de la SCE d'interaction expliquée par le modèle de régression factorielle à une covariable.

Regroupement 1

Regroupement 2

Covariable ${ }^{a *}$

Covariable $b^{* *}$
8,3

26,4
8,3

12,1
Regroupement 3

7,8

7,5
Regroupement 4

8,4

37,1

\footnotetext{
${ }^{\star}$ : déficit du nombre de grains ; ${ }^{\star \star}:$ déficit du poids de 1000 grains
}

types, ce qui concorde avec les résultats du modèle de régression conjointe. Les termes de régression sur les covariables du milieu sont moins précis que ceux du modèle de régression conjointe mais donnent des informations intéressantes (fig 5).

En ce qui concerne les coefficients de régression entre la covariable décrivant le remplissage et le rendement moyen de chaque génotype, leur observation fait apparaître 3 éléments. Premièrement, tous les coefficients sont négatifs et indiquent donc que le rendement de tous les génotypes diminue lorsque les conditions de milieu sont défavorables pendant le remplissage.
Deuxièmement, il n'y a pas de relation linéaire entre la productivité et les coefficients de régression, le coefficient de corrélation avoisinant 0,14. Un génotype peut donc associer à la fois productivité et adaptabilité à une large gamme de milieux. Ces résultats étaient déjà suggérés par l'analyse de régression conjointe. Troisièmement, les génotypes les plus stables (stabilité observée à travers l'écovalence) sont ceux pour lesquels les coefficients sont médians. Outre ces caractéristiques de stabilité et de résistance intermédiaire aux conditions défavorables de milieu, les génotypes CF9107 et VM9112 possèdent un très bon rendement moyen (calculé sur 
Fig 4. Modèle de régression conjointe : coefficients génotypiques de régression en fonction des moyennes génotypiques pour le regroupement 1. Chaque point est entouré d'une éllipse de confiance au seuil $\alpha=$ 0,05 . La droite en pointillés indique le rendement moyen. coefficients de régression

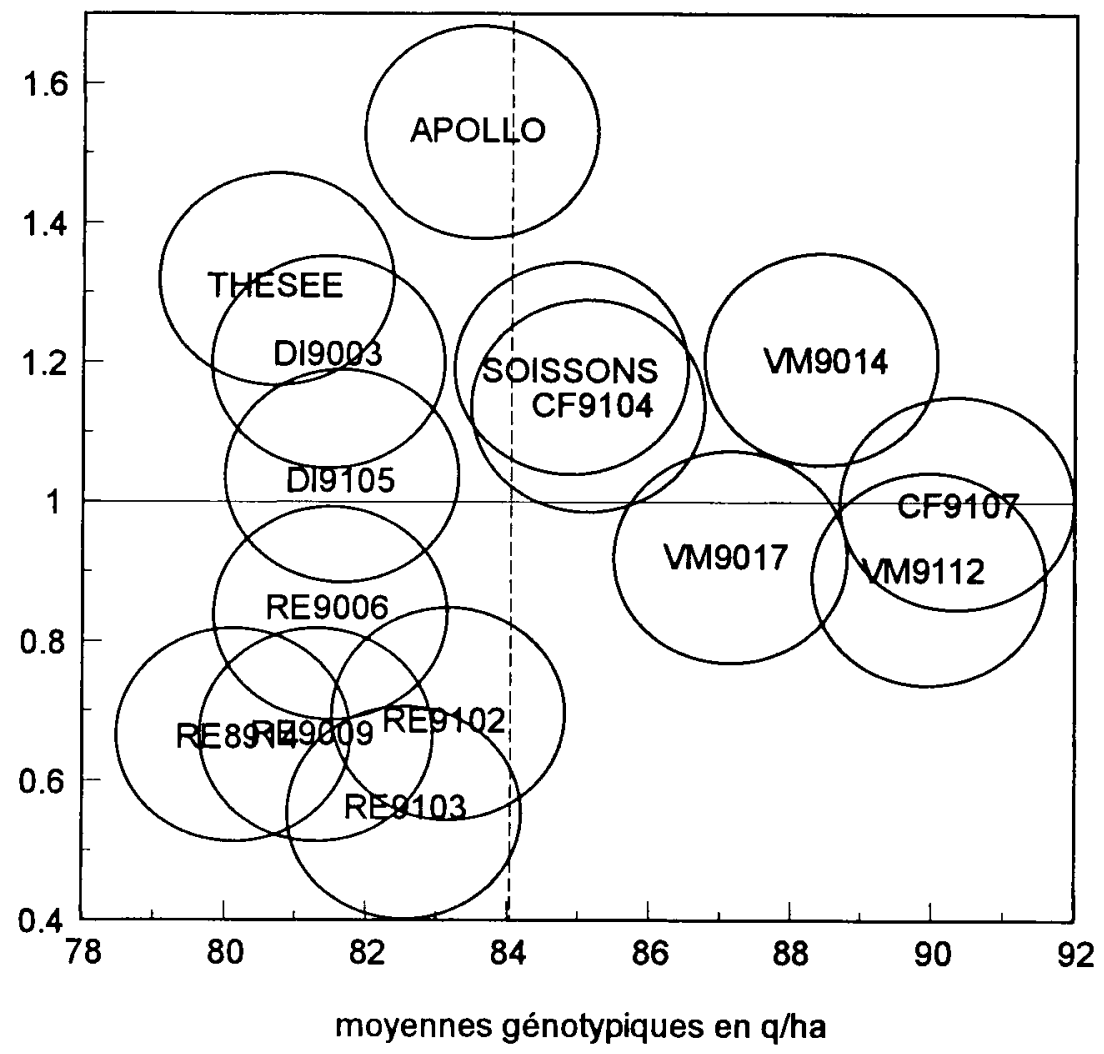

coefficients de régression

Fig 5. Modèle de régression factorielle : coefficients génotypiques de régression entre la covariable "déficit poids de 1000 grains" et le rendement en fonction des rendements génotypiques pour le regroupement 1. Chaque point est entouré d'une ellipse de confiance au seuil $\alpha=$ 0,05 . Les droites en pointillés indiquent les valeurs moyennes.

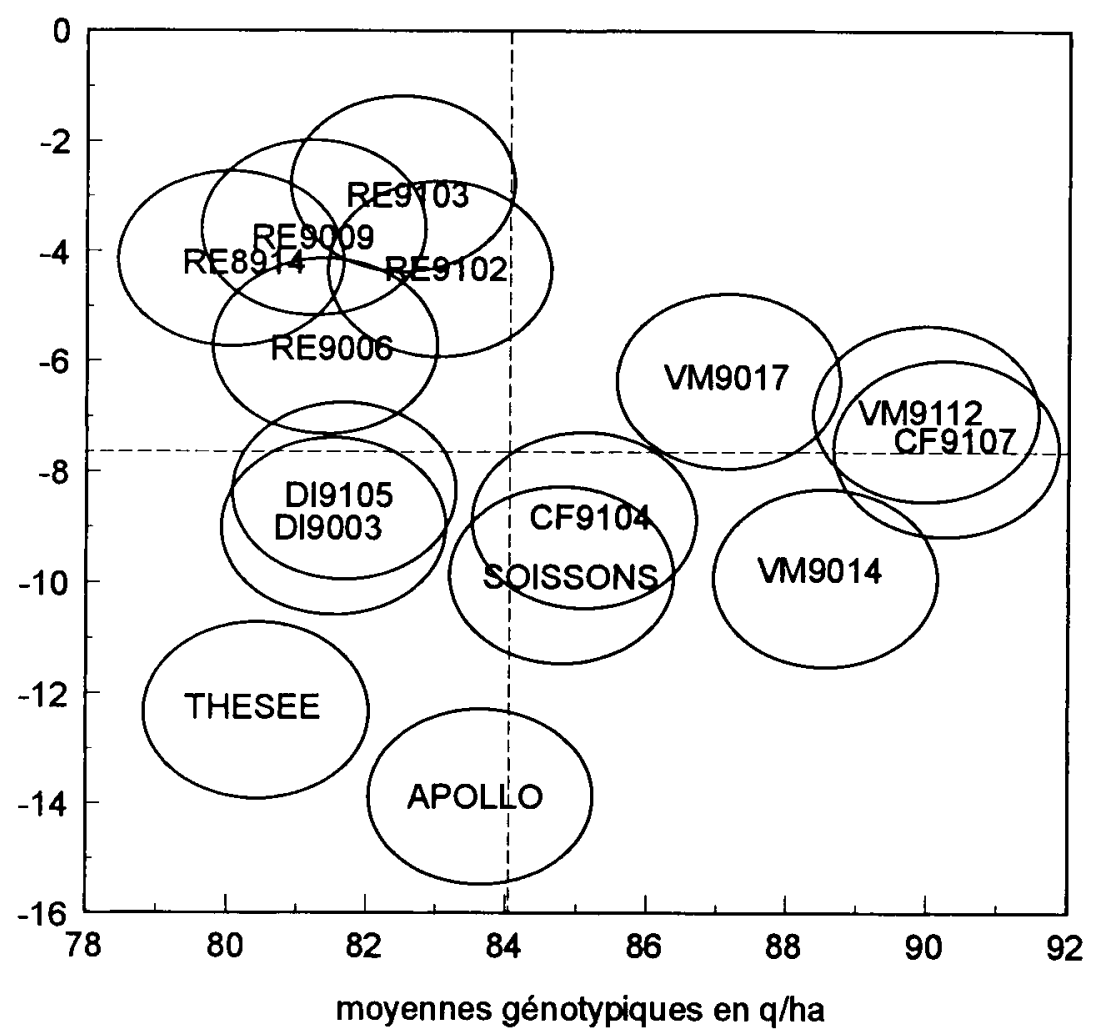


l'ensemble des milieux), largement supérieur à la moyenne de tous les génotypes dont la valeur avoisine $84 \mathrm{q} / \mathrm{ha}$.

Bien qu'en général une bonne productivité soit liée à une faible stabilité, Peltonen-Sainio et al (1993) estiment qu'il est possible de sélectionner des génotypes d'avoine à la fois productifs et stables tandis que, chez le blé tendre d'hiver, Özgen (1991) mentionne que de nouveaux génotypes à la fois productifs et adaptés à une large gamme de milieux peuvent être créés. Chez le blé tendre, nos résultats montrent qu'il est en effet possible de cumuler ces différentes caractéristiques en sélection.

Les génotypes de Rennes se démarquent des autres par leur bonne résistance aux conditions défavorables de remplissage tandis qu'Apollo et Thésée sont les plus pénalisés (fig 5). On retrouve avec ce modèle de régression factorielle les caractéristiques d'adaptation spécifique de ces génotypes définies précédemment. On peut remarquer aussi que les lignées en cours de sélection à l'INRA ont un comportement très différent de celui des témoins, ce que constatait Parisot-Baril (1992) sur du matériel issu des mêmes laboratoires.

Ce comportement très différent est dû au fait que les témoins n'ont pas été choisis alors que les lignées étudiées ont été sélectionnées pour leur productivité et leur stabilité pendant les années d'essai qui servent de base à cette étude. Les résultats obtenus sont donc un peu attendus puisqu'ils concernent le sous-ensemble du matériel qui a présenté le meilleur comportement. Cela signifie que la sélection pendant la phase d'évaluation a été efficace. Et avant cette phase d'évaluation, les schémas de sélection pratiqués à l'INRA ont été élaborés pour prendre en compte la résistance aux maladies à l'aide de pépinières non traitées aux fongicides, de plusieurs milieux de sélection et de tests de sélection pratiqués sur les jeunes générations de lignées. Les différences entre les témoins et les lignées s'expliquent donc aussi par cette phase de sélection durant les générations précoces.

Les résultats relatifs au remplissage concordent avec ceux de Baril (1992). Dans une analyse de régression factorielle faisant intervenir d'autres types de covariables du milieu, Baril a en effet montré, sur blé tendre d'hiver, que l'interaction génotype ${ }^{*}$ milieu est principalement due à des facteurs limitant le rendement durant le remplissage. En revanche, nos résultats montrent qu'il y aurait peu de variabilité entre les variétés pour la résistance aux facteurs biologiques s'exprimant pendant la phase de formation du nombre de grains. Par ailleurs, Tai (1975) a trouvé des résultats comparables chez la pomme de terre : c'est pendant la dernière phase de l'élaboration du rendement que les génotypes réagissent le plus.

L'utilisation des indicateurs du milieu permet en outre de voir que l'interaction durant le remplissage est associée à l'intensité de verse dans le milieu ainsi qu'à la pression des maladies du feuillage autres que l'oïdium et la septoriose (tableau XI). Dans le tableau XI, on constate que la covariable pression des autres maladies du feuillage explique une part importante de la SCE d'interaction seulement pour les regroupements 1 et 4 . Les différences entre les 4 regroupements sont moins importantes pour la verse. Ceci suggère que la pression parasitaire a probablement eu un impact plus fort sur le poids de 1000 grains que la verse. Ces indicateurs donnent toutefois des résultats inférieurs à ceux de Charmet et al (1993) qui expliquent, à l'aide de régression factorielle chez le ray-grass, 46 à $54 \%$ de l'inter-

Tableau XI. Pourcentage de la SCE d'interaction expliquée par le modèle de régression factorielle faisant intervenir les indicateurs environnementaux du remplissage.

\section{Covariables}

Déficit hydrique

Températures $>25^{\circ} \mathrm{C}$

Intensité de verse

Pression d'oïdium

Pression de septoriose

Pression des autres maladies du feuillage

Pression des maladies de l'épi

Pression des maladies du pied
Regroupement 1 Regroupement 2

Regroupement 3 Regroupement 4

$\begin{array}{rrrr}6,6 & 12,0 & 10,8 & \\ 9,2 & 11,5 & 14,0 & 38,0 \\ 22,7 & 19,9 & 12,2 & 37,0 \\ 6,9 & 9,8 & 10,0 & 3,7 \\ 2,9 & 7,2 & 3,1 & 15,1 \\ 36,4 & 6,7 & 5,7 & 49,4 \\ 3,2 & 5,6 & 2,9 & 27,2 \\ 5,8 & 12,7 & 11,8 & \end{array}$


action grâce à l'utilisation de 2 covariables climatiques : les températures excessives et le bilan hydrique. II faut noter cependant que la durée des phases d'élaboration du rendement est différente pour les 2 espèces. De plus, pour le blé qui possède la phase la plus longue, il existe de nombreux facteurs biotiques tels que les attaques de maladies auxquels s'ajoutent les facteurs abiotiques. Nos résultats, ainsi que ceux de Baker (1971), issus d'une analyse de covariance, et de Baril (1992) obtenus également par régression factorielle, montrent en effet que l'impact des maladies est assez important chez le blé tendre. En revanche, dans une étude en zone méditerranéenne sur blé dur, Nachit et al (1992) font intervenir d'autres variables du milieu dans leur modèle AMMl : l'altitude et l'irrigation.

\section{CONCLUSION}

L'analyse de l'interaction à l'aide des différents modèles et des regroupements permet de préciser que les lignées en cours de sélection à I'INRA ont un comportement assez différent de celui des témoins qui font partie des variétés actuelles les plus cultivées.

Les génotypes de Rennes résistent bien aux conditions défavorables de milieu, notamment durant le remplissage mais leur rendement reste limité dans les milieux très productifs et ceci n'est pas attribuable à des caractéristiques du milieu mais sans doute à des caractéristiques génotypiques. Leur comportement est inverse de celui des témoins. Ces derniers ont en effet été sélectionnés pour être cultivés selon des conduites culturales intensives avec en particulier des objectifs de rendement élevés, et une pression des maladies diminuée par une couverture fongicide et insecticide efficace. Cette étude fait donc ressortir leur bonne adaptation à ces conditions de milieu et montre qu'ils n'ont pas été sélectionnés pour des milieux à forte pression de maladies. Les génotypes de Rennes, quant à eux, sont sélectionnés dans le cadre d'un programme de recherche sur la résistance aux maladies chez le blé tendre d'hiver et le fruit de ce travail de recherche est donc illustré ici.

CF9107 et VM9112 sont à la fois des génotypes productifs, stables, présentant une bonne adaptabilité générale, ce qui laisse présager qu'il est possible d'associer rusticité et productivité en sélection. En effet, si les techniques de culture intensive pratiquées jusqu'à maintenant sont remises en cause dans le cadre de la politique agricole commune, la sélection doit maintenant concilier la recherche d'un rendement optimal avec celle de la résistance aux stress biotiques ou abiotiques.

Les milieux n'ayant pas reçu de traitement fongicide discriminent bien les génotypes résistants aux maladies des autres. Si ces milieux sont efficaces pour améliorer l'adaptation spécifique des génotypes, il faut toutefois y associer la sélection dans des milieux productifs et concilier la recherche de la stabilité avec celle de la productivité.

Le comportement très différent des témoins par rapport aux lignées est dû au fait qu'ils n'ont pas été choisis alors que les lignées étudiées ont été sélectionnées pour leur productivité et leur stabilité pendant les années d'essai qui servent de base à cette étude. II aurait sans doute été moins extrême si la totalité des lignées en cours de sélection avait pu être expérimentée au cours des 3 années d'expérimentation. C'est la limite de cette étude. En revanche, elle a permis de montrer que la sélection a été efficace pendant toute la phase d'évaluation.

Par ailleurs, le diagnostic agronomique de chaque milieu peut permettre de préciser le comportement particulier de génotypes. Certains se différencient pendant le remplissage alors que leur niveau d'interaction est comparable pendant la phase d'élaboration du nombre de grains par $\mathrm{m}^{2}$. De plus l'interaction pendant le remplissage est associée à l'intensité de verse dans le milieu ainsi qu'à la pression des maladies de feuillage autres que l'oïdium et la septoriose. Ainsi des éléments importants concernant la typologie des variétés ont pu être déterminés.

\section{RÉFÉRENCES}

Atlin GN, Frey KJ (1989) Predicting the relative effectiveness of direct versus indirect selection for oat yield in 3 types of stress environments. Euphytica 44, 137-142

Baker RJ (1971) Effects of stem rust and leaf rust of wheat on genotype-environment interaction for yield. Can J Plant Sci 51, 457-461

Baril CP (1992) Factor regression for interpreting genotype-environment interaction in bread wheat trials. Theor App/ Genet 83, 1022-1026

Becker HC, Leon J (1988) Stability analysis in plant breeding. Plant Breeding 101, 1-23

Becker HC (1981) Correlations among some statistical measures of phenotypic stability. Euphytica 30, 835-840 
Bilbro JD, Ray LL (1976) Environmental stability and adaptation of several cotton cultivars. Crop Sci 16, 821-824

Braun HJ, Pfeiffer WH, Pollmer WG (1992) Environments for selecting widely adapted spring wheat. Crop Sci 32, 1420-1427

Ceccarelli S, Grando S, Hamblin J (1992) Relationship between barley grain yield measured in low- and high-yielding environments. Euphytica $64,49-58$

Ceccarelli S (1987) Yield potential and drought tolerance of segregating populations of barley in contrasting environments. Euphytica 36, 265-273

Ceccarelli S (1989) Wide adaptation: how wide? Euphytica 40, 197-205

Charmet G, Balfourier F, Ravel C, Denis JB (1993) Genotype $x$ environment interactions in a core collection of French perennial ryegrass populations. Theor Appl Genet 86, 731-736

Decoux G, Denis JB (1991) INTERA. Logiciels pour l'interprétation statistique de l'interaction entre 2 facteurs. Laboratoire de biométrie INRA, route de Saint-Cyr, F78026 Versailles cedex, France

Denis JB (1980) Analyse de régression factorielle. Biom Praxim 20, 1-34

Denis JB (1988) Two-way analysis using covariates. Statistics 19, 123-132

Denis JB (1992) Analyse de l'interaction entre 2 facteurs. Support de cours. Laboratoire de biométrie INRA, route de Saint-Cyr, F78026 Versailles cedex, France

Eberhart SA, Russell WA (1966) Stability parameters for comparing varieties. Crop Sci 6, 36-40

Finlay KW, Wilkinson GN (1963) The analysis of adaptation in a plant-breeding programme. Aust $J$ Agric Res 14, 742-754

Freeman GH (1973) Statistical methods for the analysis of genotype-environment interactions. Heredity 31, 339-354

Gauch HG (1988) Model selection and validation for yield trials with interaction. Biometrics 44, 705-715

Gauch HG (1990) Full and reduced models for yield trials. Theor Appl Genet 80, 153-160

Hühn M (1990a) Nonparametric measures of phenotypic stability. Part 1: Theory. Euphytica 47, 189-194

Hühn M (1990b) Nonparametric measures of phenotypic stability. Part 2: Applications. Euphytica 47,195-201

Hühn M, Lolito S, Piepho HP (1993) Relationships between genotype $x$ environment interactions and rank orders for a set of genotypes tested in different environments. Theor App/ Genet 86, 943-950
Lecomte C, Hulmel M, Bernicot MH (1993) Utilisation du diagnostic agronomique pour modéliser l'interaction. In : Rapport du séminaire CTPS Techniques d'expérimentation en vue de l'évaluation des variétés, INRA, Paris, 5 mai 1993, 74-104

Lin CS, Binns MR, Lefkovitch LP (1986) Stability analysis: where do we stand? Crop Sci 26, 894-900

Lin CS, Binns MR (1988) A method of analyzing cultivar $\mathrm{x}$ location $\mathrm{x}$ year experiment: a new stability parameter. Theor App/ Genet 76, 425-430

Nachit MM, Sorrells ME, Zobel RW, Gauch HG, Fischer RA, Coffman WR (1992) Association of environmental variables with sites' mean grain yield and components of genotype-environment interaction in durum wheat. II J Genet Breed 46, 369-372

Özgen M (1991) Yield stability of winter wheat (Triticum sp) cultivars and lines. J Agric Crop Sci 166, 318-325

Parisot-Baril C (1992) Étude de la stabilité du rendement chez le blé tendre d'hiver (Triticum aestivum $L$ Thell). Thèse de doctorat de l'université Paris-Sud, $210 \mathrm{p}$

Peltonen-Sainio P, Moore K, Pehu E (1993) Phenotypic stability of oats measured with different stability analyses. J Agric Sci 121, 13-19

Perkins JM, Jinks JL (1968) Environmental and genotype-environmental components of variability. III. Multiple lines and crosses. Heredity 23, 339-356

Piepho HP, Lolito S (1992) Rank correlation among parametric and nonparametric measures of phenotypic stability. Euphytica 64, 221-225

Sage GCM, Roffey AP, Stanca AM (1984) Simultaneous selection of segregating two-row winter barley material in England and Italy. Euphytica $33,187-198$

SAS ${ }^{\circledR}$ Institute (1991) User's guide. SAS ${ }^{\circledR}$ Inst, Cary, NC, États-Unis

Simmonds NW (1991) Selection for local adaptation in a plant breeding programme. Theor Appl Genet 82 , 363-367

Tai GCC (1975) Analysis of genotype-environment interactions based on the method of path coefficient analysis. Can J Genet Cytol 17, 141-149

Westcott B (1986) Some methods of analysing genotype-environment interactions. Heredity 56, 243253

Wricke G (1962) Über eine Methode zur Erfassung der okologischen Streubreite in Feldversuchen. $Z$ Planzenzücht 47, 92-96

Yates F, Cochran WG (1938) The analysis of groups of experiments. J Agric Sci 28, 556-580 